\title{
Molecular characterization and phylogenetic analyses of virulent infectious bronchitis viruses isolated from chickens in Eastern Saudi Arabia
}

\author{
Maged Gomaa Hemida ${ }^{1,2}$, Mohammed A. Al-Hammadi ${ }^{1,} \cdot$ Abdul Hafeed S. Daleb $^{1,}$. \\ Cecilio R. Gonsalves ${ }^{1,}$
}

Received: 5 January 2017 / Accepted: 7 April 2017/Published online: 9 May 2017

(C) Indian Virological Society 2017

\begin{abstract}
Infectious bronchitis virus (IBV) is one of the major respiratory viral threats for chickens. Despite the intensive application of IBV vaccines, several outbreaks have been reported worldwide. Here, we report several IBV outbreaks in thirteen poultry farms in Eastern Saudi Arabia (ESA) from 2013 to 2014. The main goals of the current study were as follows: (1) isolation and molecular characterization of the currently circulating strains in ESA (AlHasa, Dammam, and Buqayq) and (2) evaluation of the immune status of these birds to IBV. To achieve our goals, tissue specimens (trachea, lungs, liver, kidney and cecal tonsils) and sera were collected. High morbidity up to $100 \%$ and mortality ranging from 18 to $90 \%$ were reported. Severe infection was observed in the trachea, bronchi, and kidneys of the infected birds. IBV strains were isolated using embryonated chicken eggs. The isolated viruses induced hemorrhage, dwarfing and death of the inoculated embryos 3-5 days post-infection. The circulating IBV strains were identified by sequencing the partial IBV-N and IBV-S1 genes. These viruses showed $95 \%$ sequence identity to Indian, Italian, Egyptian and Chinese strains and were quite distinct from the locally used vaccines on the genomic level. Interestingly, high antibody titers against
\end{abstract}

Electronic supplementary material The online version of this article (doi:10.1007/s13337-017-0375-7) contains supplementary material, which is available to authorized users.

Maged Gomaa Hemida

mhemida@kfu.edu.sa; gomaa55@gmail.com

1 Department of Microbiology and Parasitology, College of Veterinary Medicine, King Faisal University,

P.O. Box: 400, Al-Ahsa, Saudi Arabia

2 Department of Virology, Faculty of Veterinary Medicine, Kafrelsheikh University, Kafrelsheikh, Egypt
IBV were reported in some of these farms, suggesting the presence of new virulent strains in ESA. The seroconversion of infected birds was reported among the affected flocks. In conclusion, very virulent IBV strains are currently circulating in ESA. Further studies are currently in progress to molecularly characterize these IBV strains.

Keywords ELISA · Eastern Saudi Arabia - Infectious bronchitis virus $\cdot$ PCR

\section{Introduction}

Avian infectious bronchitis virus (IBV) is a highly contagious virus of chickens worldwide. IBV belongs to the family Coronaviridae and genus Gammacoronavirus. IBV is classified as a group 3 coronavirus [22]. The IBV genome is single-strand positive sense RNA. The viral genome organization is as follows: 5'UTR-Replicase gene (ORF1a and ORF1b)-S-M-E-N-3'UTR [22]. The nucleocapsid (N) gene is the most conserved among different coronaviruses, whereas the spike (S) glycoprotein (especially S1) is highly variable [22]. IBV was first identified in 1931 in the USA and occasional outbreaks are still reported throughout the world [2]. IBV has a wide tissue tropism, including the upper respiratory tract, urinary tract and reproductive system of chickens. IBV infection is manifested clinically in chickens in the form of respiratory distress, kidney failure, a drop in egg production and the production of malformed eggs [21]. IBV replicates in the tissues and membranes of embryonated chicken eggs, resulting in hemorrhage, dwarfing of the inoculated embryos and death in a short time after inoculation [36]. Monitoring of the immune response of different poultry flocks has been practiced using several serological 
techniques, such as hemagglutination inhibition (HI) and enzyme-linked immunosorbent assay (ELISA) [40]. Several molecular techniques were recently developed to monitor the prevalence of IBV, including gel-based PCR and realtime PCR [14]. IBV frequently undergoes changes through mutation and recombination. Mutation within the IBV-S1 gene may change viral virulence and tropism. These mutations may also affect the physicochemical and biological properties of the virus [33], resulting in the continuous emergence of new IBV strains. Conventional techniques do not cope with the rapid and frequent dynamic changes of the virus [27]. Several molecular surveys have been conducted to verify the identity of IBV strains in many regions worldwide [7, 26]. Additionally, several vaccines have been developed to protect chickens against IBV worldwide [38]. Live attenuated vaccines such as the Massachusetts strain produced good protection against several IBV strains; however, these results may be limited by the introduction of this strain to a specific area [32]. Moreover, these vaccines do not guarantee the protection of different poultry flocks against newly emergent IBV strains [32]. Currently, there is high demand for studies of different coronaviruses on the molecular level, especially in the Middle East due to the emergence of the Middle East Respiratory syndrome coronavirus (MERSCoV) [39]. We recently reported the absence of any neutralizing antibodies against MERSCoV in chicken sera from Eastern Saudi Arabia [20]. However, very few studies have been performed in Saudi Arabia to assess the prevalence of IBV, and none of these studies reported the clinical and molecular characterization of these viruses [1]. The main goal of the current study was to isolate and perform a molecular characterization of the new emergent strains of IBV in Eastern Saudi Arabia. Based on our obtained results, we report the isolation and molecular characterization of several virulent strains of IBV in ESA. Further ongoing studies are in progress in our laboratory to do full characterization of these strains. We believe this study will have great impact on our understanding of coronaviruses in the Arabian Peninsula.

\section{Materials and methods}

\section{Area of study}

Samples were collected from thirteen poultry farms in Eastern Saudi Arabia that reported clinical IBV outbreaks from 2013 to 2014 (AL-Hasa (Al-Hofuf), Dammam and Buqayq). We denote these farms as follows: AL-Hasa (AlHofof) (H), Dammam (D) and Buqayq (A). Supplementary Figure shows a Saudi map illustrating the geographic distribution of the poultry farms under study that experienced IBV outbreaks from 2013 to 2014.

\section{Sampling}

\section{Tissue specimens}

Tissues were collected from birds showing clinical IBV signs after the birds were euthanized in a carbon dioxide (10\%) chamber. Licensed veterinarians collected samples from the trachea, lungs, kidney, spleen, intestine, gizzard, proventriculus, cecal tonsil and ovaries. These tissue samples were pooled into groups of five like tissue samples in one tube. The samples were collected in the Dulbecco's modified eagle medium (DMEM) transport medium supplemented with $10 \%$ fetal bovine serum and then transported to the laboratory on ice. Tissue suspensions (10\%) were prepared as previously described [29]. All animal experiments and sample collection were performed as per The King Abdul-Aziz City of Science and Technology, Royal Decree No. M/59, (http://www.kfsh.med.sa/KFSH_ WebSite/usersuploadedfiles\%5CNCBE\%20Regulations\% 20ENGLISH.pdf). This animal utilization protocol was amended by the King Faisal's Animal Ethics and the National Committee of Bio-Ethics (NCBE).

\section{Serum samples}

A total of 370 serum samples were collected via venipuncture of the wing vein from the farms under study (Supplementary Table). Sera were separated as previously described [30]. The collected sera were heat inactivated at $56{ }^{\circ} \mathrm{C}$ for $30 \mathrm{~min}$ then stored at $-20{ }^{\circ} \mathrm{C}$.

\section{Virus isolation}

Virus was isolated from the tissue suspensions using 9-11day-old embryonated chicken eggs from native breeds (non-IBV vaccinated). The source of these eggs was free from IBV antibodies based on analysis with a commercial ELISA kit (IDEXX IBV Ab, USA). The IBV strains was isolated as previously described [10].

\section{Pathogenicity index of the circulating IBV strains in ESA}

Tissue suspensions of pooled organs from each farm were used to assess the pathogenicity indices. Approximately $100 \mu \mathrm{l}$ of each suspension representing one farm was used to inoculate five 9-day-old embryonated chicken eggs. Daily observation of the inoculated Embryonated chicken eggs (ECE) was performed by candling for up to $5 \mathrm{dpi}$. Sham PBS-inoculated eggs were also included in this experiment. Allantoic fluid and embryo processing was performed at $5 \mathrm{dpi}$ as previously described [12]. These experiments were independently repeated three times. 


\section{RNA extraction}

Viral RNA was extracted from the tissue suspensions, embryonated egg tissues and their fluids. The extraction was performed following the kit's instructions (QIAamp Viral RNA Mini Kit, Qiagen, Inc., Valencia, CA, USA). The viral RNA was stored at $-80{ }^{\circ} \mathrm{C}$.

\section{Amplification}

The oligonucleotides used to amplify the partial IBV-N, full length IBV-N and partial IBV-S1 genes are listed in Table 1.

The RT-PCR reactions were performed using the antisense strands of the oligonucleotides (Table 1) as previously described [23]. The PCR reactions were performed as previously described $[1,23]$.

\section{Sequence analysis}

The purified PCR products of the partial IBV-N and IBV$\mathrm{S} 1$ genes were sequenced in both directions. The obtained sequences were assembled into one contig. The sequences was initially analysed using the NCBI/BLAST/blastn suite (https://blast.ncbi.nlm.nih.gov/Blast.cgi?CMD=Web\&

PAGE_TYPE=BlastHome).

The accession numbers of the sequences used to develop the IBV-N and IBV-S1-based phylograms are as follows: HQ916669.1, HQ916668.1, KF663559.1, EF185916.1, FN182283.1, EU350552.1, JQ977698.1, M95169.1, FJ904722.1, X58002.1, EU812232.1, M21515.1, AY363968.1, FN182283.1, KF696629.1, KF377577.1, AF352310.1, FJ807652.1, GU393335.1, AY942745.1, GQ504721.2, AJ311362.1, GU393333.1, GU393334.1, and GU393336.1.

The phylograms were constructed using the obtained truncated IBV-N and IBV-S1 sequences. A multiple alignment was performed with the Mega-6 package using the neighbor-joining method with 1000 bootstrap replicates as previously described [35].
Enzyme-linked immunosorbent assay (ELISA)

Testing of the collected sera was performed with a commercial enzyme-linked immunosorbent assay (The IDEXX IBV Ab Test, IDEXX, USA). The ELISA procedures were performed according to the kit's instructions.

\section{Results}

\section{Clinical features of very virulent IBV outbreaks in Eastern Saudi Arabia}

Thirteen poultry farms in Eastern Saudi Arabia (12 broilers and 1 layer) experienced typical IBV infections from 2013 to 2014. Eight farms were located in Al-Hasa province (AlHofuf city, H1-H8), four farms (D1-D4) were in Dammam city and one farm was in Buqayq city (A1) (Supplementary Figure). All farms received regular commercial vaccines against IBV, NDV and IBDV, with the exception that D4 did not receive any vaccines. Chickens in some farms started to show clinical symptoms as early as 11 days of age, whereas birds in other farms showed clinical signs at 28 days. In contrast, the layer farm reported the onset of the outbreak at the 33rd week of age. High morbidity rates were reported in most of the affected farms in up to $100 \%$ of the birds. The mortality rates varied from $18 \%$ (H8 farm) to $90 \%$ (A1 farm) (Supplementary Table). The general health conditions of the birds were affected; the diseased birds exhibited ruffled feather and sometimes diarrhea (Fig. 1a). The affected birds showed typical IBV signs of respiratory distress, such as mouth breathing, gasping, rales, and nasal discharges (Fig. 1a; Supplementary movie S1). Birds within the affected farms were huddled together and concentrated around the heaters (Fig. 1b, c). Supplementary movies S1-S5 describe the progress of the IBV infection in one big commercial broiler farm in ESA. The IBV infection started from a small group of birds and then progressed quickly to include other parts of the farm, as shown in movies S1-S5. Within 6-10 days,

Table 1 List of oligonucleotides used in the current study and their information

\begin{tabular}{llll}
\hline Primer name & Sequence $5^{\prime}-3^{\prime}$ & Target gene & Referencs \\
\hline IBV-NF1 & AATTTTGGTGATGACAAGATGA & PN & Jones et al. (2011) \\
IBV-NR1 & CATTGTTCCTCTCCTCATCTG & PN & Jones et al. (2011) \\
IBV-NF2 & ATGGCGAGCGGTAAAGGATCTG & FLN & Jones et al. (2011) \\
IBV-NR2 & CAGCTGAGGTCAATGCTTTATC & FLN & Jones et al. (2011) \\
IBV-S1F1 & TAATACTGGC/TAATTTTTCAGA & PS1 & Jones et al. (2011) \\
IBV-S1R1 & AATACAGATTGCTTACAACCACC & PS1 & Jones et al. (2011) \\
\hline
\end{tabular}

$P N$ Partial IBV-N gene, PS1 partial S1, FLN Full length IBV-N gene 

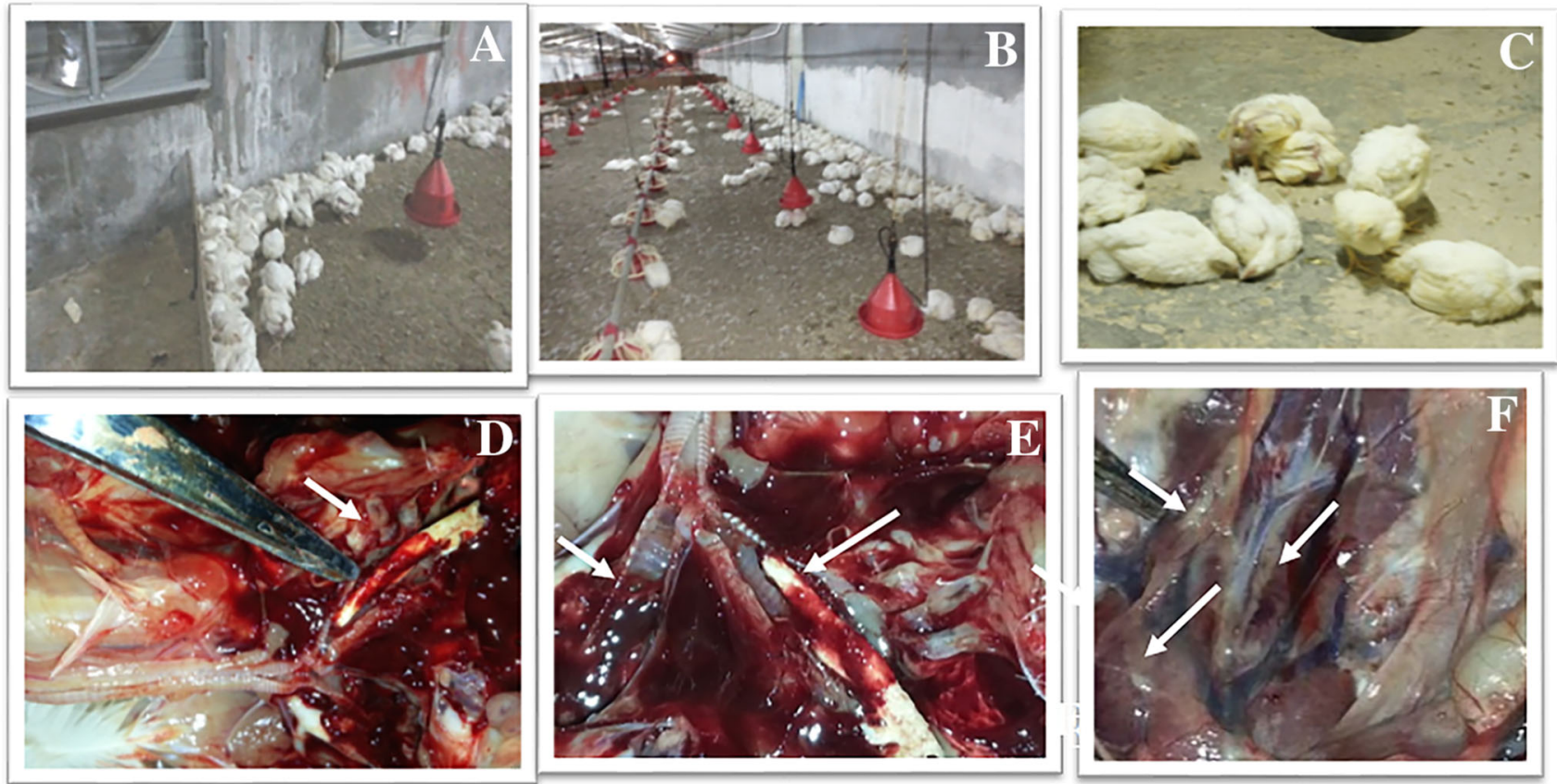

Fig. 1 Clinical profile of IBV infection in chicken farms in Eastern Saudi Arabia. An overview of the clinical picture of virulent IBV infection in poultry farms in ESA. a Birds are huddled together and collected against the source of heat beside the walls in the farm. b Very few birds are left after high mortality among the affected birds. c Close view of a group of IBV-infected birds at the morbid stage. The birds are recumbent, showing respiratory distress and

almost the entire flock became infected. Necropsy examinations of the diseased birds showing typical signs revealed congestion and hemorrhage of the upper respiratory tracts, especially the trachea and bronchi (Fig. 1d). The presence of prominent white cheesy caseous plugs in the trachea of the affected birds was observed in most of the birds during the gross pathological examination (Fig. 1d, e). In the most severely affected birds, the trachea was fully occluded with cheesy caseous plugs (Fig. 1b, c). Hemorrhage in the trachea at the bifurcation was also reported. Figure 2e shows one tracheal bifurcation of an affected bird in which one side is occluded by cheesy material and the other side is occluded by a blood clot. Kidney involvement was clearly observed in the carcasses after examination (Fig. 1f). Enlargement of the kidneys and the loss of their globular appearance was obvious (Fig. 1f).

\section{Isolation and pathogenicity index of isolated IBV strains}

The circulating IBV strains were isolated using 9-11 nonIBV vaccinated native breed embryonated chicken eggs. Briefly, the ECE were inoculated with the corresponding IBV isolates from each farm, and the allantoic fluids and embryonated tissues were harvested at 1, 3, and 5 days soiled vents. d Congestion and hemorrhage of the trachea in a bird showing a prominent caseous plug occluding the trachea of bird during the necropsy examination. e Tracheal bifurcation of one bird showing that one side of the trachea is occluded with a white cheesy caseous plug while the other side is showing a hemorrhage. $\mathbf{f}$ Kidneys of birds are enlarged in size and lose their globular appearance and the ureter is impacted with urates

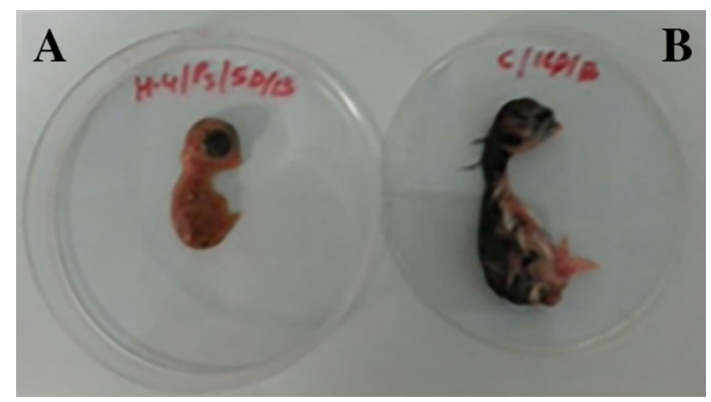

Fig. 2 Isolation of IBV using embryonated chicken eggs. Embryonated chicken eggs (9-11 days old) inoculated with IBV tissue suspensions isolated from field outbreaks in ESA. a The morphology of the IBV inoculated embryo at $5 \mathrm{dpi}$. The embryo shows dwarfism, malformation and a lack of feathers. b The PBS inoculated (sham) embryo showing the normal size and morphology with welldeveloped feather

post-inoculation. Embryos inoculated with field specimens collected from an IBV outbreak showed congestion, hemorrhage, dwarfing and death at various intervals (Fig. 2). Table 2 summarizes the pathogenicity indices of different IBV isolates using the ECE system. Most IBV-suspected samples caused embryonic death of the inoculated embryos as early as 2 days post-inoculation (dpi). By $5 \mathrm{dpi}$, most inoculated embryos had died. This result is in contrast to 
Table 2 Pathogenecity index of the isolated IBVs from farms in Eastern Saudi Arabia

\begin{tabular}{lllllllll}
\hline No & Farm & 1 dpi & 2 dpi & 3 dpi & 4 dpi & 5 dpi & Live 5 dpi & Total dead by 5 dpi \\
\hline 1 & H1 & 0 & 0 & 2 & 2 & 1 & 0 & 5 \\
2 & H3 & 0 & 0 & 2 & & 3 & 0 & 5 \\
3 & H4 & 0 & 0 & & & & 5 & 0 \\
4 & H5 & 0 & 0 & 1 & & & 4 & 1 \\
5 & H6 & 0 & 0 & & & & 5 & 0 \\
6 & H7 & 0 & 0 & 1 & 2 & & 2 & 4 \\
7 & H8 & 0 & 0 & 1 & 1 & 1 & 2 & 3 \\
8 & D1 & 0 & 0 & & 1 & 2 & 2 & 3 \\
9 & D2 & 0 & 0 & 2 & 1 & 1 & 1 & 5 \\
10 & D3 & 0 & 0 & & 2 & & 3 & 3 \\
11 & A1 & 0 & 0 & 3 & 2 & & & 5 \\
12 & Sham & 0 & 0 & & & & 5 & 0
\end{tabular}

\begin{tabular}{llllllllll}
\hline Farm/day & H-3 & H-4 & H-5 & H-6 & H-7 & H-8 & D-1 & D-2 & D-3 \\
\hline Day 1 & & $+\mathrm{Ve}$ & & $+\mathrm{ve}$ & $+\mathrm{Ve}$ & & & $+\mathrm{Ve}$ & \\
Day 3 & $+\mathrm{Ve}$ & & & & & & & & \\
Day 5 & $+\mathrm{Ve}$ & & $+\mathrm{Ve}$ & & & & $+\mathrm{Ve}$ & & $+\mathrm{Ve}$ \\
\hline
\end{tabular}

Table 3 Summary of the RTPCR results on the passage 1 of different IBV isolates from Eastern Saudi Arabia

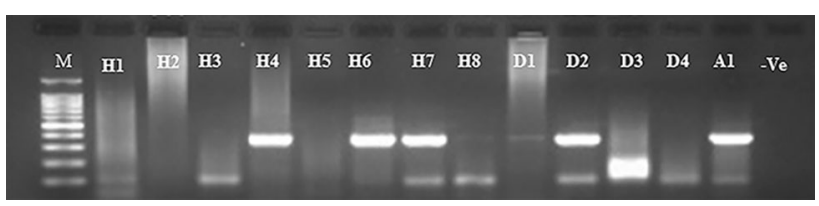

Fig. 3 Detection of IBV in embryonated chicken egg tissues and fluids. Agarose gel picture showing the amplified PCR products of the partial IBV-N gene from samples collected from the pathogenicity index studies. Samples from embryos and their fluids were tested for the presence of IBV at different intervals after egg inoculation. Lane (M) DNA marker, $1 \mathrm{~kb}$ ladder, Lanes (H1-H8) represent RT-PCR results of samples collected from farms in Al-Hasa (Farm-1-Farm 8), Lanes (D1-D4) showing results of samples collected from farms in Dammam (D1-D4), Lane (A1) showing result of samples collected from farm in Abqaia region. Lane $(-\mathrm{Ve})$ showing non template negative control

conduct this molecular surveillance. The truncated IBV$\mathrm{N}$ primers amplified $260 \mathrm{nt}$ and the truncated IBV-S1 primer amplified $313 \mathrm{nt}$. IBV was detected in the pooled organs from each farm (Fig. 3). Both primers showed consistent results for the detection of the IBV isolated in this survey; however, the IBV-S1 primers showed higher specificity than the IBV-N primers (Fig. 4). To verify the tropism of the isolated IBV strains, IBV amplicons were detected in different body organs (trachea, lung, kidney, spleen, gizzard, proventriculus, intestine, cecal tonsils and pancreas) by RT-PCR as previously described (Fig. 5).
We conducted molecular-based surveillance of IBV among the 13 farms in ESA. We used two primers to 


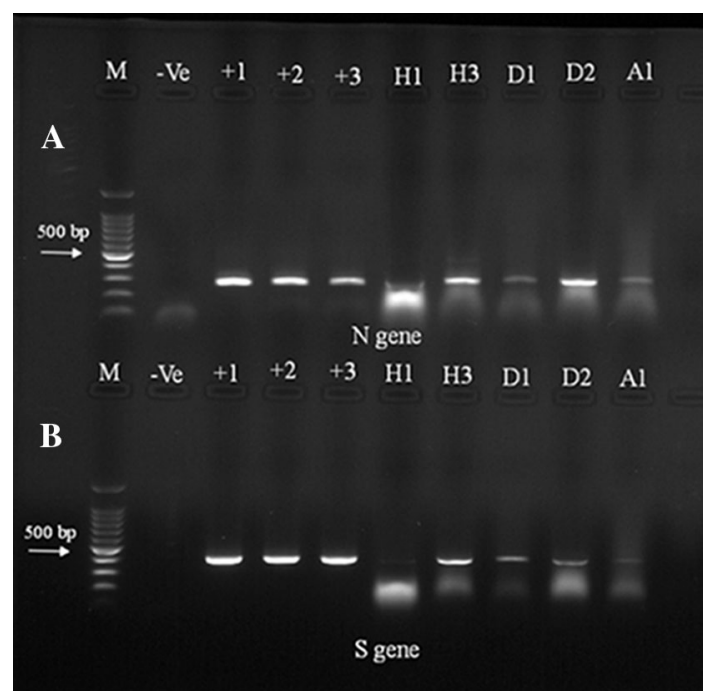

Fig. 4 Molecular-based prevalence of IBV in ESA by gel-based RTPCR. Agarose gel electrophoresis picture showing the RT-PCR results of tested specimens from farms in Alhasa (H1and $\mathrm{H} 3$ ), Dammam (D1and D2) and one farm from Abqaiq (A1). The size of the amplified amplicons by using the partial IBV-N primer $(220 \mathrm{nt}$, upper panel) and the partial IBV-S1 primer (330 nt, lower panel). Lane (M) 100 bp DNA marker, Lanes 1-3 (IBV vaccines). Lane $(-\mathrm{Ve})$ non DNA template negative control

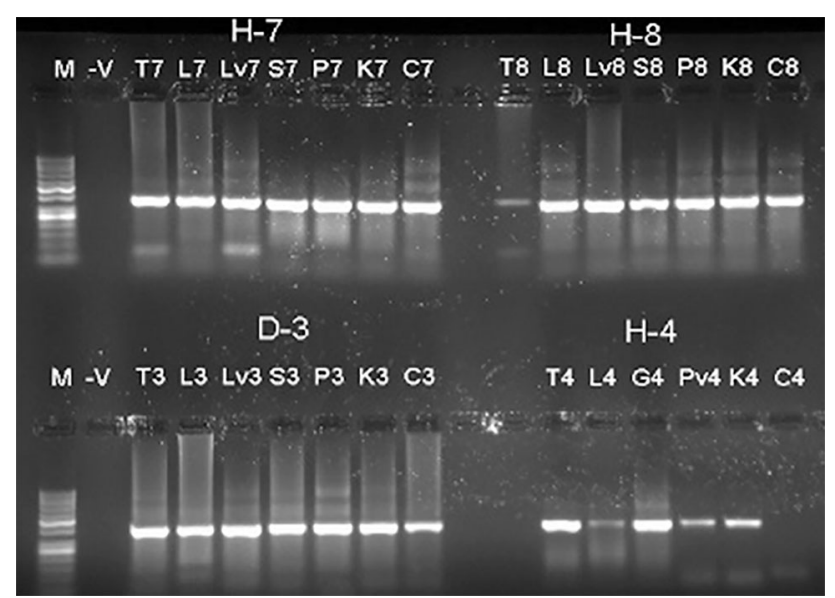

Fig. 5 Detection of IBV in different body organs. Agarose gel picture showing the amplified PCR products of the partial IBV-N from samples collected from IBV-infected chickens. Lane (M): DNA ladder $1 \mathrm{~kb}$. Results of tested organs from (T7, L7, S7, P7, K7, and C7) are showing pooled samples from trachea (T), lung (L), spleen $(\mathrm{S})$, proventriculus $(\mathrm{P})$, kidney $(\mathrm{K})$, and cecal tonsils $(\mathrm{C})$. Lane $(-\mathrm{V})$ representing non-template DNA was included as a negative control

\section{The currently circulating IBV strains in Eastern Saudi Arabia are different from the locally used vaccine strains}

Our results showed that the isolated IBV strains did not exhibit positive amplification when tested using the IBV-N full-length gene oligonucleotides based on the currently used vaccine in Eastern Saudi Arabia (Fig. 6).

\section{Phylogenetic analysis of isolated IBV from Eastern Saudi Arabia}

The partial IBV-N and IBV-S1 gene sequences reported in this study were deposited in Genbank and assigned accession numbers KT762154, and KU358908, respectively. Blasting these sequences against the Genbank revealed high nucleotide identity to other IBV and TCoV strains from Israel, South Africa, and South America (95, 94, and 93\%, respectively). Both the IBV-N and IBV-S1based phylograms showed close relationships to other IBV strains from India, China, Egypt, and Israel (Figs. 7 and 8) (Table 4).

\section{Seroprevalence of IBV in the Eastern region of KSA}

A total of 370 serum samples were tested for the presence of IBV antibodies using the commercially available IBV ELISA kits. According to the obtained results, 298 (80\%) samples were positive and 72 samples (20\%) were negative (Table 5). We developed a scoring system for the positive samples based on the concentration of IBV antibodies in the sera of tested birds. For example, out of the 19 samples tested from farm $\mathrm{H} 3$, five were negative and $14(73 \%)$ were positive $(+)$. The details of the serosurveillance of the selected farms are listed in Table 5.

\section{Discussion}

IBV is an endemic viral disease of poultry in many regions of the world [11], including the. Middle East [2]. Little is known about the molecular prevalence of IBV in the Gulf area, especially Saudi Arabia. Although intensive IBV vaccination is currently practiced in most poultry populations throughout the Kingdom, many outbreaks are still reported [1]. These outbreaks may be due to the massive and random use of IBV vaccines. Another possibility is the emergence of new IBV strains resulting from the frequent mutations and recombination between several IBV strains [1]. The main goals of the current study were to isolate the currently circulating IBV strains in ESA, to use molecular methods to assess the prevalence among the affected farms and to evaluate the immune status of the chickens in those farms against IBV. From 2013 to 2014, several chicken farms (12 broilers and 1 layer) reported IBV infections in ESA. The affected farms showed variable mortality rates that ranged from 15\% (H8) to 90\% (A1) (Supplementary Table). High mortality rates due to IBV infection were reported in many parts in the world, especially with the 


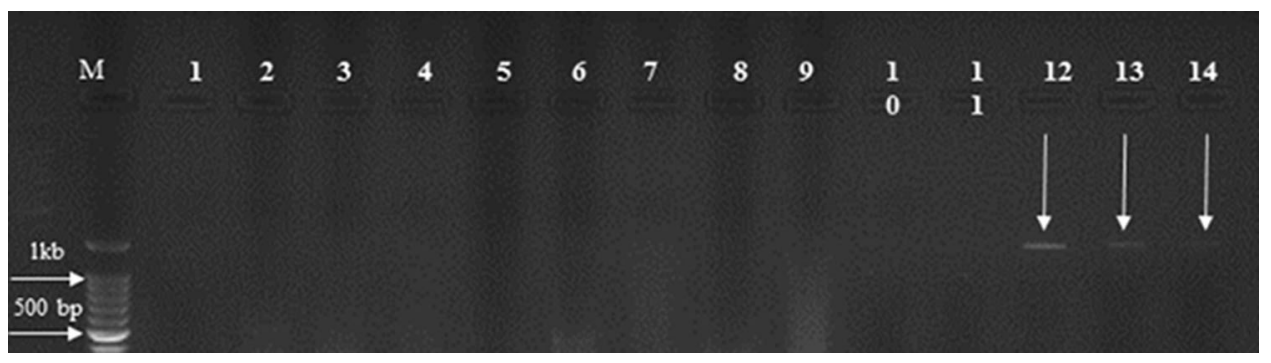

Fig. 6 IBV isolated from ESA is different from common vaccine strains. Agarose gel picture showing full length IBV-N gene amplification. Lane M: DNA marker, $1 \mathrm{~kb}$, lanes $1-11$ show IBV confirmed samples by partial IBV-N gene primers showing no amplicons using the full length IBV gene. Lanes 12-14 showing positive amplification in the the full length IBV-N gene for three common commercial IBV vaccines (H120 and IBV-4-91, respectively)
Fig. 7 Phylogenetic analysis based on the partial IBV-N sequences of the isolated IBV from Eastern Saudi Arabia. Phylogenetic tree based on the obtained IBV-N partial sequences from IBV-infected farms in Eastern Saudi Arabia. The neighbor-joining tree was constructed from the truncated IBV-N gene alignment with the other IBV-V sequences in Genbank. IBV isolated from Eastern Saudi Arabia clustered with other IBV strains from Egypt, India, and China as well as other members of group 3 coronaviruses such as the beluga whale coronavirus and duck coronavirus

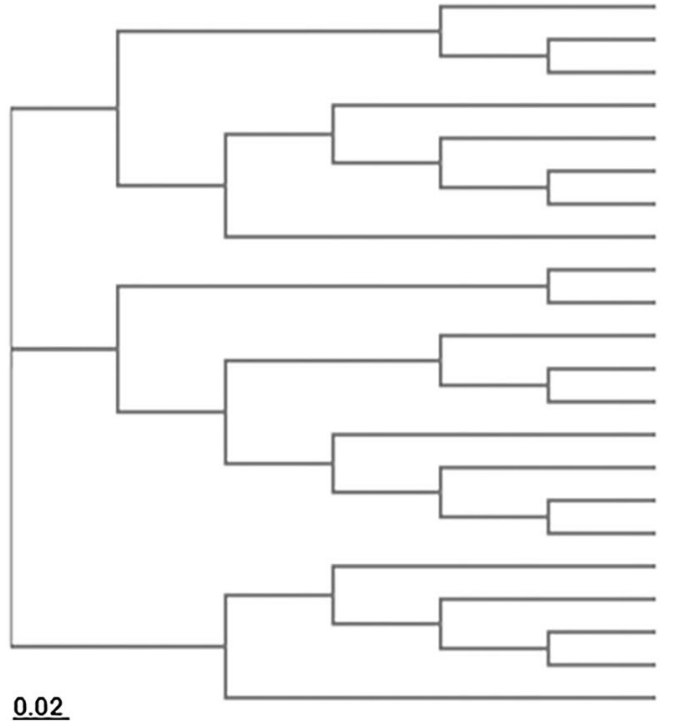

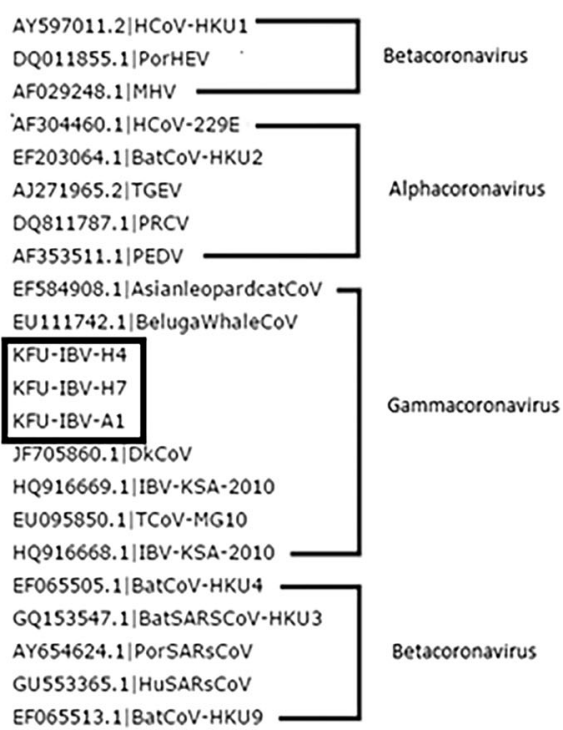

emergence of new strains of the virus [11, 15, 16, 34]. Moreover, experimental detection of IBV infections in vaccinated chicken flocks has recently been reported [37]. In the current study, 12 out of 13 farms received regular IBV vaccines and other recommended vaccines for Newcastle disease virus (NDV) and infectious bursal disease virus (IBDV) ( Supplementary Table). Only farm D4 did not receive any vaccines to IBV or any other viruses; the owner of this farm believed that vaccination was not of great help in protecting his birds against IBV. In response to this outbreak, we collected tissue samples (trachea, lung, kidney, intestine, gizzards, proventriculus, and pancreas) and sera from these farms. The affected birds showed clinical symptoms that included coughing, sneezing, rales, nasal discharge, white diarrhea, dullness, huddling together, and sudden death in acute cases. Here, we report the progression of IBV infections in some chicken farms in ESA. First, a few birds became infected and showed signs of rapid breathing, loss of appetite, and loss of body weight gain (Supplementary movie 1; Fig. 1a-c). The infection spread throughout the flock as more birds were affected. An increase in the reported daily mortality was also observed (Supplementary movies 2 and 3). Within 6-10 days, most of the birds in certain farms became infected. The mortality reports reached their peak approximately 5-6 days post-infection. By the 10th day from the onset of the infection, most of the birds were dead. The chicken pen was found to be almost empty with the exception of a very few birds scattered in the moribund stage (Supplementary movie 4, Fig. 1a-c).

Necropsy examination of birds in the morbid stage revealed congested trachea, occluded bronchi with white cheesy caseous plugs, congested lungs, swollen kidneys with a loss of lobulation, impacted ureters, and vent pasting with white diarrhea (Fig. 1d-f).

ECE is a good system for IBV isolation [18]. Tissue suspensions from each farm were inoculated into 9-11day-old embryonated (non-vaccinated native breed) eggs from IBV-free Baladi birds. The source of these eggs was demonstrated to be IBV-free using a commercial IBV 


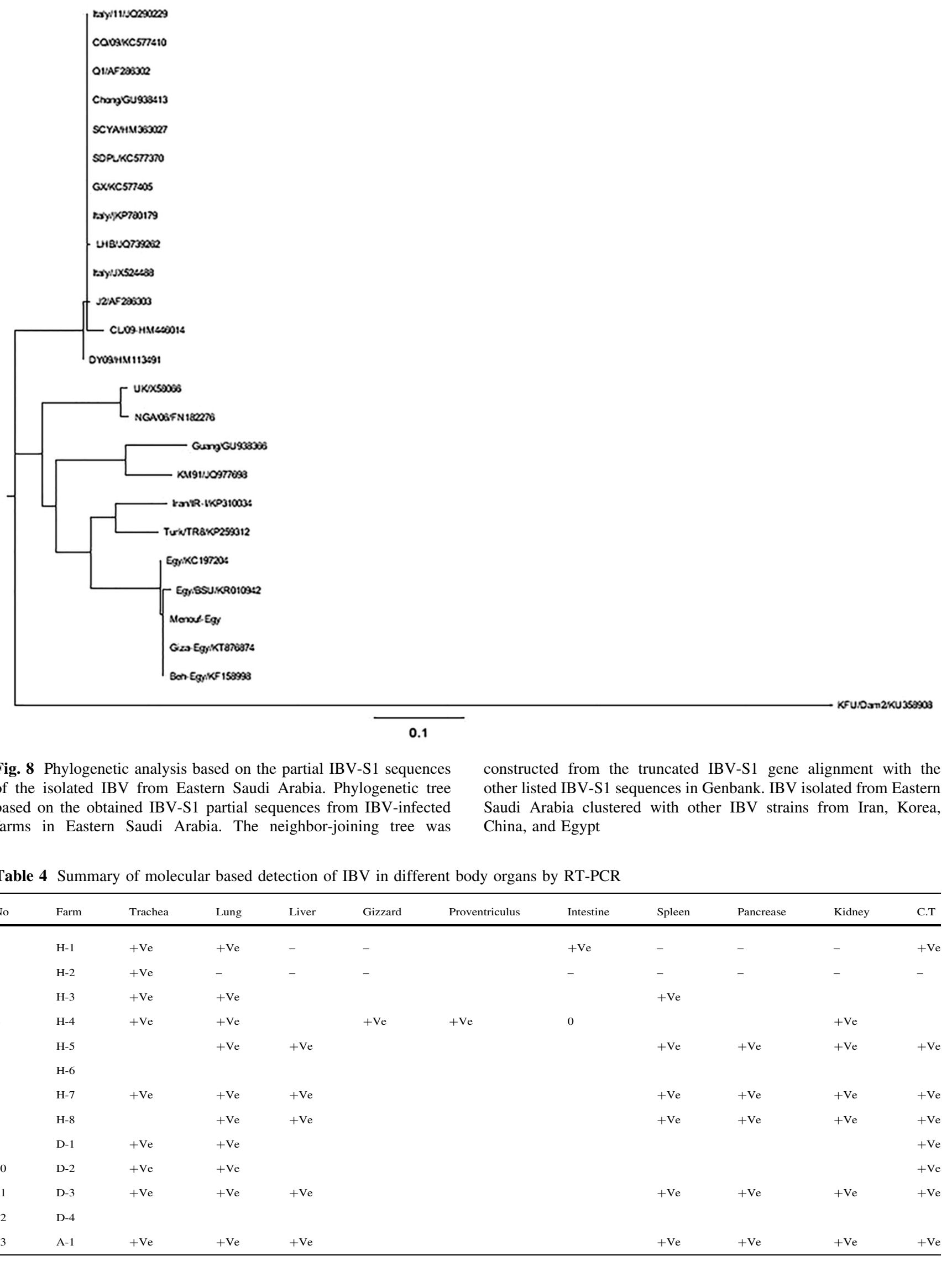


Table 5 Seroprevalence of IBV in some chicken farms in Eastern Saudi Arabia

\begin{tabular}{|c|c|c|c|c|c|c|c|c|c|}
\hline \multirow[t]{2}{*}{ Farm } & \multirow[t]{2}{*}{ Total tested } & \multirow[t]{2}{*}{$(-\mathrm{Ve})$} & \multirow[t]{2}{*}{$(+\mathrm{Ve})$} & \multirow[t]{2}{*}{$\%(+\mathrm{Ve})$} & \multicolumn{5}{|c|}{$(+\mathrm{Ve})$ scores } \\
\hline & & & & & + & ++ & +++ & ++++ & +++++ \\
\hline $\mathrm{H}-3$ & 19 & 5 & 14 & 73 & 14 & & & & \\
\hline H-5 & 74 & 25 & 49 & 66 & 40 & 9 & & & \\
\hline $\mathrm{H}-7$ & 47 & 8 & 39 & 82 & 25 & 10 & 4 & & \\
\hline $\mathrm{H}-8$ & 77 & 7 & 70 & 90 & 60 & 10 & & & \\
\hline D-1 & 24 & 15 & 9 & 37 & 9 & & & & \\
\hline D-2 & 26 & 5 & 21 & 80 & 20 & 1 & & & \\
\hline D-3 & 63 & 7 & 56 & 88 & 46 & 7 & 3 & & \\
\hline D-4* & 40 & 0 & 40 & 100 & 1 & 8 & 11 & 9 & 11 \\
\hline Total & 370 & 72 & 298 & 80 & 215 & 45 & 18 & 9 & 11 \\
\hline
\end{tabular}

D4 farm did not received any IBV vaccines
ELISA kit (data not shown). The isolated IBV induced hemorrhage, dwarfing, curling and death of the ECE 3-5 dpi (Fig. 2). The inoculated materials, including embryos and their fluids, were isolated 5 dpi. Then, the ECE was used to determine the pathogenicity indices of the IBV strains as previously described [8]. IBV isolates from farms $\mathrm{H} 1, \mathrm{H} 3, \mathrm{H} 7$ and $\mathrm{A} 1$ were highly pathogenic and produced $100 \%$ mortality among the inoculated ECE by 5 dpi (Table 3). In contrast, the IBV isolates from farms $\mathrm{H} 5, \mathrm{H} 8$, and D2 were moderately pathogenic and induced approximately $50 \%$ mortality among the inoculated eggs, and isolates from farms H4, H6, D1 and D3 exhibited low pathogenicity and did not induce any deaths or marked effects on the inoculated eggs. All embryos survived until the end of the experiment. These experiments were independently repeated three times to ensure that our data were solid. We also tested for the presence of IBV in the allantoic fluid and tissues of the inoculated ECE by RTPCR using the IBV-N primers (Table 3 ). We were able to detect IBV nucleic acids in samples from farms H4, H6, H7 and D2 at 1 dpi. Only H3 was positive for IBV by RT-PCR at 3 dpi. However, samples from the eggs inoculated with isolates from farms $\mathrm{H} 3 \mathrm{H} 5, \mathrm{D} 1$ and $\mathrm{D} 3$ were positive up to 5 dpi (Table 3; Fig. 3). Both gel-based PCR and real-time PCR were previously used to test different samples for IBV $[4,14]$. We used gel-based RT-PCR to conduct molecularbased prevalence testing among the poultry farms under study. We used this approach to detect the tropism of different IBV isolates by detecting the presence of IBV nucleic acids in different tissues collected from the diseased birds. Our results showed positive IBV signals from all 13 tested farms (Fig. 4a, b). For instance, the trachea, lung, intestine, and cecal tonsil samples from farm $\mathrm{H} 1$ were positive, as shown in Table 4 and Fig. 4a, b. However, only samples from farm $\mathrm{H} 4$ showed a positive reaction in the gizzard and proventriculus of the tested birds. Interestingly, samples from several farms showed positive reactions in the pancreas, such as $\mathrm{H} 5, \mathrm{H} 7, \mathrm{H} 8, \mathrm{D} 3$ and $\mathrm{A} 1$.
The most prevalent positive reactions among most farms were in the trachea, lung, spleen kidneys and cecal tonsils (Table 4; Fig. 4). These data suggested that the isolated IBV strains were pantropic viruses because they had the potential to replicate in many organs of several body systems. Interestingly, the clinical picture of these IBV isolates in chickens was very similar to that of the Middle East respiratory syndrome coronavirus (MERSCoV). MERS$\mathrm{CoV}$ was recently demonstrated to induce lower respiratory tract infections in the lungs as well as kidney failure [41]. This phenomenon may be due to the adoption of mechanisms by the coronavirus to induce pathology in the affected host [41]. Interestingly, we recently reported the absence of MERSCoV antibodies in chicken sera in Eastern Saudi Arabia [20]. IBV vaccination failure has been previously reported [31]. There are many reasons for the vaccination failures, such as immunosuppressive agents (IBDV infection, Marek's disease virus, Cryptosporidium or Eimeria infections), inadequate vaccine dosage, and the use of vaccines prepared from strains different from the circulating strains $[5,9,13,25]$. Our results showed that the currently circulating IBV strains in ESA were quite distinct from the commonly used vaccine in this area. Figure 7 shows the absence of any amplicons of the IBV strains isolated from ESA in an assay based on three different vaccines. These results suggest the presence of new virulent IBV strains circulating in ESA. The continuous reports of IBV outbreaks may be at least partially due to the use of non-homologous vaccine strains. Indeed, frequent recombination was previously reported among different IBV strains in cases of multiple infections [17]. The obtained IBV-N sequences were used to construct the neighborhood-joining phylogram (Fig. 7). Our results showed that the isolated IBV strains belonged to the avian coronaviruses group (gamma coronaviruses) along with the turkey coronavirus, duck coronavirus and beluga whale coronavirus [19]. Similar results were obtained using a partial IBV-S1 gene from the isolated strains (Fig. 8). 
These isolates were closely related to other IBV strains from Italy, the Middle East and Asia [1, 3, 9, 28]. Most local farms are currently using the Mas-H120 vaccine for regular IBV vaccination. Based on the obtained partial IBV-N and IBV-S1 sequences, the local isolated strains show $79 \%$ nucleotide identity to the Mass-H120 vaccine strains (Figs. 7, 8). This result implies that these poultry producers need to revise their vaccination programs. Vaccines from the currently circulating strains should be prepared and used for their vaccination programs.

Several approaches have been used to detect antibodies against IBV in the sera of different birds [6]. We used a commercial ELISA kit to evaluate the immune status of chickens in poultry farms under investigation for IBV. Although all farms in this study were vaccinated against IBV except D4, we were able to detect antibodies in the sera from all 13 farms including D4 (Table 5). The presence of antibodies in the sera of the test birds in farm D4 in the absence of any vaccine against IBV suggested that these birds seroconverted in response to IBV infection. According to our data, 298 (80\%) birds showed positive antibodies against IBV, whereas 72 birds (20\%) were antibody negative (Table 5). Furthermore, we designed a scoring system based on the antibody concentration in a given sample. Briefly, serum samples with S/P ratios less than 0.2 were considered negative, from 0.2 to $0.9(+)$, from 1 to $1.9(++)$, from 2 to $2.9(+++)$, from 3 to $3.9(++++)$ and more than 4 $(+++++)$. Interestingly, we found 11 birds from farm D4 that were $(+++++)$ although this farm did not administer an IBV vaccine. This result suggested that active field infection triggered very high antibody production among the infected birds (Table 5). Farm H3 (showing 60\% mortality) had 14 birds with only a (+) antibody concentration, which might be due to the partial neutralization of the effect of active field infection. Perhaps the presence of antibodies against IBV in the sera of chickens in association with field infection due to the emergence of new IBV strains does not affect the action of the vaccine administered to the birds. To the best of our knowledge, this is the first study reporting the isolation and molecular characterization of very virulent IBV strains in Eastern Saudi Arabia.

Acknowledgements This work was supported by the King Faisal University Deanship of research Grant No. (142017). We also than Mr. Ahmed Alkhars with his laboratory technical helps during the virus isolation and pathogenicity index.

\section{References}

1. Ababneh M, Dalab AE, Alsaad S, Al-Zghoul M. Presence of infectious bronchitis virus strain CK/CH/LDL/97I in the Middle East. ISRN Vet Sci. 2012;2012:201721. doi:10.5402/2012/ 201721.
2. Abdel-Moneim AS, El-Kady MF, Ladman BS, Gelb J. S1 gene sequence analysis of a nephropathogenic strain of avian infectiousbronchitis virus in Egypt. Virology. 2006;3:78.

3. Abdel-Moneim AS, Zlotowski P, Veits J, Keil GM, Teifke JP. Immunohistochemistry for detection of avian infectious bronchitis virus strain M41 in the proventriculus and nervous system of experimentally infected chicken embryos. Virol J. 2009;6:15. doi:10.1186/1743-422X-6-15.

4. Acevedo AM, Perera CL, Vega A, Rios L, Coronado L, Relova D, et al. A duplex SYBR Green I-based real-time RT-PCR assay for the simultaneous detection and differentiation of Massachusetts and non-Massachusetts serotypes of infectious bronchitis virus. Mol Cell Probes. 2013;27(5-6):184-92. doi:10.1016/ j.mcp.2013.06.001.

5. Atkins KE, Read AF, Walkden-Brown SW, Savill NJ, Woolhouse ME. The effectiveness of mass vaccination on Marek's disease virus (MDV) outbreaks and detection within a broiler barn: a modeling study. Epidemics. 2013;5(4):208-17. doi:10.1016/j.epi dem.2013.10.001.

6. Bronzoni RV, Fatima M, Montassier S, Pereira GT, Gama NM, Sakai V, et al. Detection of infectious bronchitis virus and specific anti-viral antibodies using a Concanavalin A-SandwichELISA. Viral Immunol. 2005;18(3):569-78. doi:10.1089/vim. 2005.18.569.

7. Chacon JL, Rodrigues JN, Assayag Junior MS, Peloso C, Pedroso AC, Ferreira AJ. Epidemiological survey and molecular characterization of avian infectious bronchitis virus in Brazil between 2003 and 2009. Avian Pathol. 2011;40(2):153-62. doi:10.1080/ 03079457.2010.544641.

8. Choi KS, Lee EK, Jeon WJ, Park MJ, Kim JW, Kwon JH. Pathogenicity and antigenicity of a new variant of Korean nephropathogenic infectious bronchitis virus. J Vet Sci. 2009;10(4):357-9.

9. de Geus ED, van Haarlem DA, Poetri ON, de Wit JJ, Vervelde L. A lack of antibody formation against inactivated influenza virus after aerosol vaccination in presence or absence of adjuvantia. Vet Immunol Immunopathol. 2011;143(1-2):143-7. doi:10.1016/ j.vetimm.2011.05.023.

10. De Wit JJ. Detection of infectious bronchitis virus. Avian Pathol. 2000;29(2):71-93. doi:10.1080/03079450094108.

11. Dhama K, Singh SD, Barathidasan R, Desingu PA, Chakraborty $\mathrm{S}$, Tiwari R, et al. Emergence of Avian Infectious Bronchitis Virus and its variants need better diagnosis, prevention and control strategies: a global perspective. Pak J Biol Sci. 2014;17(6):751-67.

12. Dhinakar Raj G, Suresh Kumar K, Nainar AM, Nachimuthu K. Egg: embryo weight ratio as an indicator of dwarfism induced by infectious bronchitis virus. Avian Pathol. 2004;33(3):307-9. doi:10.1080/0307945042000205883.

13. Eladl AH, Hamed HR, Khalil MR. Consequence of Cryptosporidiosis on the immune response of vaccinated broiler chickens against Newcastle disease and/or avian influenza. Vet Res Commun. 2014;38(3):237-47. doi:10.1007/s11259-0149610-5.

14. Fan WQ, Wang HN, Zhang Y, Guan ZB, Wang T, Xu CW, et al. Comparative dynamic distribution of avian infectious bronchitis virus M41, H120, and SAIBK strains by quantitative real-time RT-PCR in SPF chickens. Biosci Biotechnol Biochem. 2012;76(12):2255-60.

15. Fellahi S, El Harrak M, Ducatez M, Loutfi C, Koraichi SI, Kuhn $\mathrm{JH}$, et al. Phylogenetic analysis of avian infectious bronchitis virus S1 glycoprotein regions reveals emergence of a new genotype in Moroccan broiler chicken flocks. Virol J. 2015;12:116. doi:10.1186/s12985-015-0347-8.

16. Feng J, Hu Y, Ma Z, Yu Q, Zhao J, Liu X, et al. Virulent avian infectious bronchitis virus, People's Republic of China. Emerg 
Infect Dis. 2012;18(12):1994-2001. doi:10.3201/eid1812. 120552.

17. Gelb J Jr, Wolff JB, Moran CA. Variant serotypes of infectious bronchitis virus isolated from commercial layer and broiler chickens. Avian Dis. 1991;35(1):82-7.

18. Guy JS. Isolation and propagation of coronaviruses in embryonated eggs. Methods Mol Biol. 2015;1282:63-71. doi:10.1007/ 978-1-4939-2438-7_7.

19. Hemida Maged Gomaa. Barta JR, Ojkic D, Yoo D. Complete genomic sequence of turkey coronavirus. Virus Res. 2008;135(2): 237-46. doi:10.1016/j.virusres.2008.03.020.

20. Hemida MG, Perera RA, Wang P, Alhammadi MA, Siu LY, Li M, et al. Middle East Respiratory Syndrome (MERS) coronavirus seroprevalence in domestic livestock in Saudi Arabia, 2010-2013. Euro Surveill. 2013;18(50):20659.

21. Ignjatovic J, Ashton DF, Reece R, Scott P, Hooper P. Pathogenicity of Australian strains of avian infectious bronchitis virus. J Comp Pathol. 2002;126(2-3):115-23. doi:10.1053/jcpa. 2001.0528.

22. Jackwood MW, Boynton TO, Hilt DA, McKinley ET, Kissinger JC, Paterson AH, et al. Emergence of a group 3 coronavirus through recombination. Virology. 2010;398(1):98-108. doi:10. 1016/j.virol.2009.11.044.

23. Ji J, Xie J, Chen F, Shu D, Zuo K, Xue C, et al. Phylogenetic distribution and predominant genotype of the avian infectious bronchitis virus in China during 2008-2009. Virol J. 2011;8:184. doi:10.1186/1743-422X-8-184.

24. Jones RM, et al. Development and validation of RT-PCR tests for the detection and $\mathrm{S} 1$ genotyping of infectious bronchitis virus and other closely related gammacoronaviruses within clinical samples. Transbound. Emerg. Dis. 2011;58(5):411-20.

25. Kim JK, Kayali G, Walker D, Forrest HL, Ellebedy AH, Griffin YS, et al. Puzzling inefficiency of H5N1 influenza vaccines in Egyptian poultry. Proc Natl Acad Sci U S A. 2010;107(24): 11044-9. doi:10.1073/pnas.1006419107.

26. Li M, Wang XY, Wei P, Chen QY, Wei ZJ, Mo ML. Serotype and genotype diversity of infectious bronchitis viruses isolated during 1985-2008 in Guangxi. China Arch Virol. 2012;157(3): 467-74. doi:10.1007/s00705-011-1206-6.

27. Lim TH, Lee HJ, Lee DH, Lee YN, Park JK, Youn HN, et al. An emerging recombinant cluster of nephropathogenic strains of avian infectious bronchitis virus in Korea. Infect Genet Evol. 2011;11(3):678-85. doi:10.1016/j.meegid.2011.01.007.

28. Mahmood ZH, Sleman RR, Uthman AU. Isolation and molecular characterization of Sul/01/09 avian infectious bronchitis virus, indicates the emergence of a new genotype in the Middle East. Vet Microbiol. 2011;150(1-2):21-7. doi:10.1016/j.vetmic.2010. 12.015 .
29. Mardani K, Noormohammadi AH, Hooper P, Ignjatovic J, Browning GF. Infectious bronchitis viruses with a novel genomic organization. J Virol. 2008;82(4):2013-24.

30. Reichmann KG, Barram KM, Brock IJ, Standfast NF. Effects of regular handling and blood sampling by wing vein puncture on the performance of broilers and pullets. Br Poult Sci. 1978; 19(1):97-9. doi:10.1080/00071667808416448.

31. Roh HJ, Hilt DA, Williams SM, Jackwooda MW. Evaluation of infectious bronchitis virus Arkansas-type vaccine failure in commercial broilers. Avian Dis. 2013;57(2):248-59. doi:10.1637/ 10459-112812-Reg.1.

32. Sharma JM. Introduction to poultry vaccines and immunity. Adv Vet Med. 1999;41:481-94.

33. Shen S, Law YC, Liu DX. A single amino acid mutation in the spike protein of coronavirus infectious bronchitis virus hampers its maturation and incorporation into virions at the nonpermissive temperature. Virology. 2004;326(2):288-98. doi:10.1016/j.virol. 2004.06.016.

34. Spickett AM, De Klerk D, Enslin CB, Scholtz MM. Resistance of Nguni, Bonsmara and Hereford cattle to ticks in a Bushveld region of South Africa. Onderstepoort J Vet Res. 1989;56(4): 245-50.

35. Tamura K, Stecher G, Peterson D, Filipski A, Kumar S. MEGA6: molecular evolutionary genetics analysis version 6.0. Mol Biol Evol. 2013;30(12):2725-9. doi:10.1093/molbev/mst197.

36. Tarpey I, Orbell SJ, Britton P, Casais R, Hodgson T, Lin F, et al. Safety and efficacy of an infectious bronchitis virus used for chicken embryo vaccination. Vaccine. 2006;24(47-48):6830-8. doi:10.1016/j.vaccine.2006.06.040..

37. Toro H, Pennington D, Gallardo RA, van Santen VL, van Ginkel $\mathrm{FW}$, Zhang J, et al. Infectious bronchitis virus subpopulations in vaccinated chickens after challenge. Avian Dis. 2012;56(3):5018. doi:10.1637/9982-110811-Reg.1.

38. Yan F, Zhao Y, Hu Y, Qiu J, Lei W, Ji W, et al. Protection of chickens against infectious bronchitis virus with a multivalent DNA vaccine and boosting with an inactivated vaccine. J Vet Sci. 2013;14(1):53-60.

39. Zaki AM, van Boheemen S, Bestebroer TM, Osterhaus AD, Fouchier RA. Isolation of a novel coronavirus from a man with pneumonia in Saudi Arabia. N Engl J Med. 2012;367(19):181420. doi:10.1056/NEJMoa1211721.

40. Zhang J, Guo Y, Xiao Y, Wang X, Li Z, Hu S, et al. A simple and rapid strip test for detection of antibodies to avian infectious bronchitis virus. J Vet Med Sci. 2010;72(7):883-6.

41. Zhao G, Jiang Y, Qiu H, Gao T, Zeng Y, Guo Y, et al. Multi-organ damage in human dipeptidyl peptidase 4 transgenic mice infected with middle east respiratory syndrome-coronavirus. PLoS ONE. 2015;10(12):e0145561. doi:10.1371/journal.pone.0145561. 